\title{
Co-existence of Congenital Adrenal Hyperplasia and Familial Hypokalemic Periodic Paralysis due to CYP21A2 and SCN4A Pathogenic Variants
}

\author{
(D) Tuğba Kontbay1, (D) Ihsan Turan ${ }^{1,2}$
}

1Şanlıurfa Training and Research Hospital, Clinic of Pediatric Endocrinology, Şanlıurfa, Turkey

${ }^{2}$ Çukurova University Faculty of Medicine, Department of Pediatric Endocrinology, Adana, Turkey

What is already known on this topic?

Pathogenic variants in the CYP21A2 gene are the most common cause of congenital adrenal hyperplasia. Familial hypokalemic periodic paralysis (FHPP) is a rare disorder in which affected individuals may experience paralytic episodes associated with hypokalemia caused by pathogenic variants in $S C N 4 A$ and CACNA1S. It is important to obtain a careful and detailed medical history from the patient and family and to identify family members at risk by segregation analyses.

\section{What this study adds?}

This is the first case report of 21-hydroxylase enzyme deficiency (21-OHD) and FHPP in the literature. Elevated adrenocorticotropic hormone and androgens may trigger a hypokalemia attack in FHPP. It is necessary to reconsider routine fludrocortisone treatment in children with 21-OHD based on additional, newly available scientific evidence.

\begin{abstract}
Steroid 21-hydroxylase deficiency is the most common cause of congenital adrenal hyperplasia (CAH), usually due to biallelic variants in CYP21A2. Classical 21-hydroxylase deficiency is characterised by virilisation of the external genitalia in females and hypocortisolism. Hyponatremia and hyperkalemia are among the common biochemical findings. Familial hypokalemic periodic paralysis (FHPP) is a rare disorder in which affected individuals may experience paralytic episodes associated with hypokalemia, caused by pathogenic variants in SCN4A or CACNA1S. A 14-year-old female, who had been diagnosed with classical 21-hydroxylase deficiency and treated with hydrocortisone and fludrocortisone since early infancy, presented with acute onset weakness. The laboratory results revealed a remarkably low serum potassium level. The family history revealed that both her father and uncle had the same hypokalemic symptoms, which suggested an FHPP diagnosis. We found two previously reported homozygous variants in the CYP21A2 (p.Ile173Asn) and SCN4A (p.Arg672His) genes in the patient. Therefore, diagnoses of simple virilising 21-hydroxylase deficiency and FHPP were genetically confirmed. Here, FPHH and chronic overtreatment with fludrocortisone may explain the presentation of our patient with severe hypokalemia. The family's medical history, which is always a valuable clue, should be investigated in detail since rare inherited conditions may co-occur in geographies where consanguineous marriages are common and the genetic pool is diverse. In patients with $\mathrm{CAH}$, care should be taken to avoid overtreatment with fludrocortisone. Androgens may have triggered the hypokalemic attack in FHPP, as supported in a previous study.
\end{abstract}

Keywords: CYP21A2, familial hypokalemic periodic paralysis, $S C N 4 A$, congenital adrenal hyperplasia

Address for Correspondence: İhsan Turan MD, Çukurova University Faculty of Medicine, Department of Pediatric Endocrinology, Adana, Turkey

Phone: +90 5333604146 E-mail: ihsanturan@hotmail.com ORCID: orcid.org/0000-0002-5654-247X
Conflict of interest: None declared Received: 18.09 .2020 Accepted: 17.12 .2020 


\section{Introduction}

Autosomal recessive pathogenic variants in the gene encoding CYP21A2 cause 21-hydroxylase enzyme deficiency (21-OHD), which is the most common reason for pediatric adrenal insufficiency, resulting in hyponatremia and hyperkalemia. The incidence ranges from 1:13,000 to $1: 15,000$ births, and the prevalence varies according to ethnicity and geographic area (1). Familial hypokalemic periodic paralysis (FHPP) is associated with pathogenic variants in genes encoding ion channel subunits. These include heterozygous pathogenic variants which may occur in the CACNA1S gene or the SCN4A gene. About $40-60 \%$ of FHPP cases are attributed to $C A C N A 1 S$ pathogenic variants, whereas in $7-10 \%$ of the cases, $S C N 4 A$ pathogenic variants are responsible. FHPP is an uncommon cause of transient episodes of painless muscle weakness due to low serum potassium levels, with an estimated prevalence of 1 in 100,000 . The disorder is less common in women. Paralytic attacks can be triggered by high-carbohydrate meals, exercise, stress, and certain medications. The mechanism that leads to episodic potassium transfer into the cells and causes weakness due to calcium channel defect is not precisely understood. The initial symptoms occur during the first or second decades of life, and these attacks vary in frequency and duration (2). To our knowledge, this is the first case report of the co-existence of FHPP with 21-OHD, which causes a reversed pathology in blood potassium levels.

\section{Case Report}

A 14-year-old female presented with acute onset weakness in both upper and lower extremities, which had progressed over a period of one day. The medical history revealed that she was diagnosed with 21-OHD as a new-born and was given oral hydrocortisone and fludrocortisone treatment. The patient was born at term in another primary care centre. There was a consanguineous marriage between her parents. Unfortunately, we could not retrieve detailed data on the relevant laboratory parameters before her referral to our clinic.

She presented with clitoromegaly, electrolyte imbalance, elevated adrenocorticotropin hormone (ACTH) and 17-OH progesterone levels. To the best of our knowledge ACTH stimulation tests were not performed. She had undergone clitoroplasty in infancy. Six months before a hypokalemic attack, while she was taking hydrocortisone $\left(17 \mathrm{mg} / \mathrm{m}^{2} /\right.$ day-irregularly) and fludrocortisone (0.1 mg/day) treatment, her total testosterone (1.03 ng/mL), 17-OH progesterone (162 ng/mL) and ACTH (190 pg/mL) levels were elevated, reflecting poor control. The patient had developed central precocious puberty with advanced bone age and received gonadotrophin releasing hormone analogue treatment until 11 years of age.

She presented to the pediatric emergency clinic with severe generalised weakness in both arms and legs, which was our first evaluation of her. There was no history of fever, acute gastroenteritis, substance abuse or carbohydrate-rich meal or exercise before the paralysis episodes. She had no history of recent upper respiratory tract infection or vomiting, and she did not experience any neurological symptoms. She did not report similar episodes in the past, but her father and uncle had a history of paralytic episodes accompanied by hypokalemia without a genetic diagnosis. The muscle strength in both arms and legs was at grade two, and reflexes were absent. Blood pressure was 100/70 mmHg. The rest of the physical examination was unremarkable. Complete blood count, blood gas, blood urea nitrogen, creatinine, creatine phosphokinase, and serum electrolytes (calcium, phosphate, magnesium) were in the normal ranges, while serum potassium and sodium levels were $1.9 \mathrm{mEq} / \mathrm{L}$ and $146 \mathrm{mmol} / \mathrm{L}$, respectively. Hyperthyroidism was excluded.

She was admitted to the hospital, fludrocortisone treatment was terminated, intravenous potassium replacement was initiated. When the lower limit of the normal serum potassium level was achieved, intravenous potassium treatment was stopped, and oral potassium (citrate and bicarbonate) administration was initiated. Oral potassium was preferred to intravenous treatment to reduce the risk of rebound hyperkalemia and discontinuation of the normal serum potassium level. Cardiac activity and serum electrolyte levels were monitored during and after the treatment. Potassium levels were closely checked for potential rebound hyperkalemia for 24 hours. In her first endocrinology evaluation, 15 days after the hypokalemic attack and without fludrocortisone treatment, her laboratory examinations showed normal plasma aldosterone concentration of $36 \mathrm{ng} / \mathrm{dL}(4-58 \mathrm{ng} / \mathrm{dL})$ and normal plasma renin activity (PRA) of $2.38 \mathrm{ng} / \mathrm{mL} / \mathrm{hr}(2.3-37.0 \mathrm{ng} / \mathrm{mL} /$ hr) with normal blood pressure. The levels of biochemical parameters of the patient are shown in Table 1.

The patient's clinic and laboratory findings and family history were consistent with FHPP. Although the patient's father had the same diagnosis, FHPP, genetic analysis had not been performed before. Blood samples for genetic analyses were obtained after informed consent was received from the patient and her parents. Sanger sequencing in the proband revealed a homozygous p.Ile173Asn (c.518T > A) pathogenic variant in CYP21A2 (NM_000500.9). The patient's mother and father were heterozygous for this variant. No pathogenic variant was detected in CACNA1S. The sequencing of SCN4A 


\begin{tabular}{|c|c|c|c|c|c|c|c|c|}
\hline $\begin{array}{l}\text { Time } \\
\text { according to } \\
\text { hypokalemic } \\
\text { attack }\end{array}$ & $\begin{array}{l}\mathrm{Na} \mathrm{mmol} / \mathrm{L} \\
(136-146)\end{array}$ & $\begin{array}{l}\mathrm{K} \mathrm{mEq} / \mathrm{L} \\
(3.5-5.5)\end{array}$ & $\begin{array}{l}\text { Total } \\
\text { testosterone } \\
\mathrm{ng} / \mathrm{dL} \\
(2.3-13.9)\end{array}$ & $\begin{array}{l}17-\mathrm{OH} \\
\text { progesterone } \\
\text { ng/mL } \\
(0.4-12)\end{array}$ & $\begin{array}{l}\text { ACTH } \\
\mathrm{pg} / \mathrm{mL} \\
(10-60)\end{array}$ & $\begin{array}{l}\text { Aldosterone } \\
\text { ng/dL (4-58) }\end{array}$ & $\begin{array}{l}\text { PRA ng/ } \\
\mathrm{mL} / \mathrm{hr} \\
(2.3-37.0)\end{array}$ & $\begin{array}{l}\text { Blood pressure } \\
\mathrm{mm} / \mathrm{Hg}\end{array}$ \\
\hline $\begin{array}{l}\text { Six months } \\
\text { before }\end{array}$ & 141 & 4.2 & 133 & 53 & 67 & NA & NA & Normotensive* \\
\hline $\begin{array}{l}\text { Three months } \\
\text { before }\end{array}$ & 142 & 4.5 & 103 & 162 & 190 & NA & NA & Normotensive* \\
\hline Days of attack & 146 & 1.9 & NA & NA & NA & NA & NA & $100 / 70$ \\
\hline 15 days' after & 143 & 4.6 & NA & NA & NA & 36.8 & 2.38 & $90 / 60$ \\
\hline
\end{tabular}

*: Blood pressure value unspecified, reported normotensive in data, numbers in parentheses refer to normal value. PRA: plasma renin activity, NA: not available, ACTH: adrenocorticotropin hormone

(NM_000334.4) in the proband identified the previously reported heterozygous p.Arg672His (c.2015G > A) variant. Her affected father and uncle were heterozygous for this variant. Two variants were considered to be 'pathogenic' variants based on the 2015 American College of Medical Genetics/Association for Molecular Pathology (ACMG/AMP) guideline. Pedigree and segregation analyses are shown in Figure 1 .

\section{Discussion}

Here, we present the first patient with co-existence of congenital adrenal hyperplasia $(\mathrm{CAH})$ and hypokalemic periodic paralysis. This is a rare case that presented with hypokalemia and a tendency to hyperkalemia due to 21 OHD.

In CYP21A2, a T-to-A change at nucleotide 518 was predicted to be a substitution of the isoleucine at residue 173 for asparagine, p.Ile173Asn. This pathogenic variant has been widely reported in 21-OHD, and functional analysis has been performed $(3,4)$. The p.Arg672His was located at a hotspot region in SCN4A. The missense variant was not found in gnomAD. Alternative variants Arg672Ser, Cys, Gly and Ser have been reported previously (5). Jurkat-Rott et al (6) reported a family with hypokalemic periodic paralysis type 2 , in which the family member had p.Arg672His in SCN4A. Lastly, this variant was considered to be a 'pathogenic' variant based on the 2015 ACMG/AMP guideline. These findings led us to conclude that this variant is the diseasecausing variant.

Since enzyme activity is $1-5 \%$ in patients with p.lle173Asn in CYP21A2, most patients do not need fludrocortisone treatment. The presence of homozygous p.Ile173Asn genotype, classified in group c, predicts simple virilising 21 OHD. However, these patients tend to have hyperkalemia $(3,4)$. Fludrocortisone treatment is initiated before the definite diagnosis, as suggested in $\mathrm{CAH}$ guidelines because
CYP21A2: p.I173N SCN4A: p. R672H

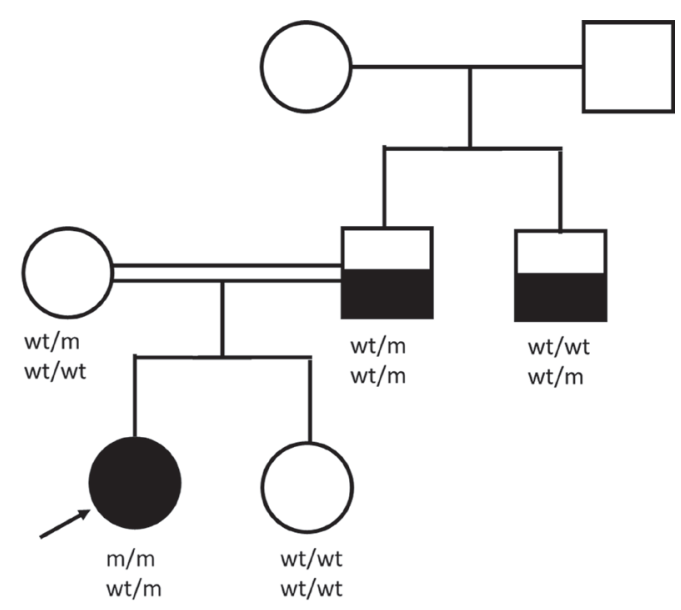

Figure 1. Affected males (Familial Hypokalemic Periodic Paralysis) are represented by black and white squares, affected, females (two diseases) are represented by black circles, and index individuals are indicated by arrows. White square symbols indicate unaffected male family members, White circle symbols represent unaffected female family members, and the double line indicates consanguinity. Under each symbol are the genotypes with WT and M denoting wildtype and mutant, respectively. The first line, and the second line indicates CYP21A2:p.I173N, and SCN4A:p.R672H, respectively

of its life-threatening nature, causing $25 \%$ of patients to receive unnecessary fludrocortisone treatment (1). It is necessary to measure PRA and aldosterone levels, blood pressure and serum electrolytes of each patient with 21 OHD to avoid overtreatment or mistreatment. We observed that this management was insufficient in the patient's history.

Additionally, genetic analysis is guiding in these patients $(3,7)$. Prior to deciding on such treatment, it is vital to review all possibilities, take a careful medical history and family history and perform a physical examination. Here, CYP21A2 genetic analysis is also highly useful in deciding upon fludrocortisone treatment (3). It was unusual to detect hypokalemia with a sign of hyperaldosteronism in 
a case where we expected a possible lack of aldosterone production. However, the patient's family history was suspicious for hypokalemic periodic paralysis. We determined that genetic analysis had not been performed for any family members before, although molecular genetic confirmation of the possible diagnosis is extremely helpful in such complex situations. In this patient, genetic testing confirmed an autosomal dominant SCN4A-related disorder and a simple virilising form of 21-OHD. Fludrocortisone treatment was discontinued gradually, with the molecular genetic prediction, as it could exacerbate possible episodes of hypokalemia. As research in upcoming fields, such as molecular genetics and metabolomics, increases, more evidence-based approaches may be created rather than empirical treatments. This provides a better medical approach to orphan cases. In our patient, when the serum potassium level was 1.9 serum $\mathrm{mEq} / \mathrm{L}$, the serum sodium level was $146 \mathrm{mmol} / \mathrm{L}$, at the upper end of the range. Additionally, 15 days after the hypokalemic attack, PRA seemed to be suppressed at the lower end of the range without fludrocortisone treatment. This finding led us to infer that mistreatment with fludrocortisone may have aggravated hypokalemia.

When patients are evaluated after or between attacks, the diagnosis of FHPP may be challenging. Molecular genetic testing is recommended when the diagnosis of FHPP is suspected (2). Other diagnostic options include provocative testing and electromyography. Provocative testing with oral glucose, insulin, exercise and ACTH can induce FHPP (8). The father and uncle of the case, carrying the same variant in SCNN4, had experienced their first hypokalemia attack in their 20s. Although there is an opinion that penetrance is lower in women, our case had her first attack at the age of 14 years. Hypokalemia that occurs earlier than we expected could be due to two reasons; first, based on the ACTH provocation test, the elevated ACTH, due to poor metabolic disease control, may trigger the attack or second, elevated androgens due to 21-OHD may trigger an attack in FHPP, as shown in the study of Ke et al (9).

Our patient had undergone clitoroplasty in infancy, without any complications. The association between malignant hyperthermia and hypokalemia is uncertain, but has been described previously (10). If surgery with general anaesthesia is required, patients should be monitored for signs of malignant hyperthermia. However, in a future surgical operation, the surgeon, anaesthesiologist and endocrinologist should evaluate possible risks and take the necessary precautions. Simultaneously, the patient was warned about drugs and general anaesthesia that could trigger an attack. In this patient's clinical management, with two co-existing conditions, we advised her to have a lowcarbohydrate diet and to refrain from strenuous exercise to prevent hypokalemic attacks. However, potassium-sparing treatment is not preferred for the risk of hyperkalemia. It can, paradoxically, worsen hypokalemia. As soon as symptoms begin, we recommend that hypokalemia must be confirmed with blood tests before potassium therapy. The patient was educated about the regular use of glucocorticoid therapy to avoid poor metabolic control with $\mathrm{CAH}$.

In medical evaluation, it is essential to take a careful and detailed patient medical history and obtaine the same from family members. The pedigree is critical. Many patients are not diagnosed, and their diseases are not defined due to insufficient examination time and clinical attention, as well as a lack of medical information, especially in orphan patients. This is the group of patients who suffer most from the difficulties in getting appropriate support. According to our clinical experience from these cases, identifying individuals at risk with segregation analysis after taking a complete family history will keep the case safer in terms of future risky situations.

\section{Conclusion}

In our case, a disease predisposing to hypokalemia, 21OHD, was suspected at the first evaluation, and molecular genetic tests supported the diagnosis. Periodic paralysis is a rare disease, and the possibility of concomitant 21-OHD is extremely low. Rare inherited conditions may co-occur in geographies where consanguineous marriage is common and the genetic pool is diverse. Due to the heavy patient load, insufficient number of doctors and the presence of widespread routine algorithms, such rare cases can be missed. In this case, our experiences highlighted once again, the importance of obtaining a careful and detailed family medical history, which allows the clinician to reduce the possible risks. Family members at risk should be identified by segregation analysis in inherited genetic diseases and disorders. Androgens may have triggered the hypokalemic attack in FHPP, as previously suggested, and overtreatment of fludrocortisone may have aggravated hypokalemia.

\section{Ethics}

Informed Consent: The subject and her parents have given their written informed consent to publish their case in accordance with the Declaration of Helsinki.

Peer-review: Externally peer-reviewed.

\section{Authorship Contributions}

Surgical and Medical Practices- Concept - Design - Data Collection or Processing - Analysis or Interpretation Literature Search - Writing: Tuğba Kontbay, İhsan Turan. 
Financial Disclosure: The authors declared that this study received no financial support.

\section{References}

1. Speiser PW, Arlt W, Auchus RJ, Baskin LS, Conway GS, Merke DP, Meyer-Bahlburg HFL, Miller WL, Murad MH, Oberfield SE, White PC. Congenital adrenal Hyperplasia due to steroid 21-hydroxylase deficiency: an endocrine society clinical practice guideline. J Clin Endocrinol Metab 2018;103:4043-4088.

2. Statland JM, Fontaine B, Hanna MG, Johnson NE, Kissel JT, Sansone VA, Shieh PB, Tawil RN, Trivedi J, Cannon SC, Griggs RC. Review of the diagnosis and treatment of periodic paralysis. Muscle Nerve 2018;57:522-530. Epub 2017 Nov 29

3. Turan I, Tastan M, Boga DD, Gurbuz F, Kotan LD, Tuli A, Yuksel B. 21-Hydroxylase deficiency: mutational spectrum and genotypephenotype relations analyses by next-generation sequencing and multiplex ligation-dependent probe amplification. Eur J Med Genet 2020;63:103782. Epub 2019 Oct 2

4. Speiser PW, Dupont J, Zhu D, Serrat J, Buegeleisen M, Tusie-Luna MT, Lesser M, New MI, White PC. Disease expression and molecular genotype in congenital adrenal hyperplasia due to 21 -hydroxylase deficiency. J Clin Invest 1992;90:584-595.
5. Kopanos C, Tsiolkas V, Kouris A, Chapple CE, Albarca Aguilera M, Meyer R, Massouras A. VarSome: the human genomic variant search engine. Bioinformatics 2019;35:1978-1980.

6. Jurkat-Rott K, Mitrovic N, Hang C, Kouzmekine A, Iaizzo P, Herzog J, Lerche H, Nicole S, Vale-Santos J, Chauveau D, Fontaine B, LehmannHorn F. Voltage-sensor sodium channel mutations cause hypokalemic periodic paralysis type 2 by enhanced inactivation and reduced current. Proc Natl Acad Sci USA 2000;97:9549-9554.

7. Riedl S, Rohl FW, Bonfig W, Bramswig J, Richter-Unruh A, FrickeOtto S, Bettendorf M, Riepe F, Kriegshauser G, Schonau E, Even G, Hauffa B, Dorr HG, Holl RW, Mohnike K, Group ACS. Genotype/ phenotype correlations in 538 congenital adrenal hyperplasia patients from Germany and Austria: discordances in milder genotypes and in screened versus prescreening patients. Endocr Connect 2019;8:86-94.

8. Streeten DH, Speller PJ, Fellerman H. Use of corticotropin-induced potassium changes in the diagnosis of both hypo- and hyperkalemic periodic paralysis. Eur Neurol 1993;33:103-108.

9. Ke Q, Luo B, Qi M, Du Y, Wu W. Gender differences in penetrance and phenotype in hypokalemic periodic paralysis. Muscle Nerve 2013;47:41-45. Epub 2012 Sep 27

10. Lambert C, Blanloeil Y, Horber RK, Berard L, Reyford H, Pinaud M. Malignant hyperthermia in a patient with hypokalemic periodic paralysis. Anesthesia and Analgesia 1994;79:1012-1014. 